\section{PSQ-139 ORTHOPAEDIC IMPLANT RESUPPLY CHAIN: BETTER, FASTER, STRONGER!}

M Kayal* ${ }^{*}$ C Moafo, L Bergua, S Bergon. Centre Hospitalier Eure Seine- Hôpital D'evreux, Seine-Maritime, Evreux, France

10.1136/ejhpharm-2019-eahpconf.572

Background The 58 trauma compositions, including surgical instruments and sterilisable orthopaedic implants, are often sent incomplete to the central sterilisation (CS) concerning the implants. Sterilising incomplete compositions or keeping them at the CS until they are completed, leads to patient risks such as postponing or delaying surgery.

Purpose This study aimed at quantifying the incomplete compositions, the costs and suggestion of solutions with a multidisciplinary commission (MC).

Material and methods During 3 months, the sterilisation technicians (ST) counted how many times the compositions were sent incomplete. The costs were based on the surgical instrument number per composition, the checking complexity and the employee, water and electricity costs. Surgeon, nurse, pharmacist and STs reviewed the results and the existing implant resupply chain.

Results Eighty-four per cent of the orthopaedic compositions were sent incomplete. Either the nurse had forgotten to send the implants to the CS on time for $42 \%$ of them or the order had not yet been delivered. The delivery delay differed from 3 to 10 days. One-third of the compositions stayed more than a day at the CS before being completed. Sterilising incomplete composition cost $€ 1156$ for 3 months. The checking by ST could last 1 hour $30 \mathrm{~min}$ to make sure all the implants were present. The MC concluded to switch from sterilisable implants to sterile implants at an equivalent cost, and to substitute the biomedical service for the pharmacy to make order.

Conclusion With the pharmacy, the delay delivery shortened to 48 hour. The company provided the sterile devices freely, which were paid for when implanted. Misusing once-only use implants was avoided thanks to sterilisable patterns helping select the right implants. The removal of the incomplete composition sterilisation costs offset the sterile implant packaging elimination costs. The composition simplification saves time for the checking by the STs and makes the composition available quicker for the operating room (OR). Patient safety is improved thanks to a permanent and computerised implant traceability which also automatically makes an order once done. However, the switch implies a reorganisation in the OR's storage facility.

This new optimised implant resupply chain ensures safety for the OR and the patients, and cost effectiveness for the hospital.

\section{REFERENCES AND/OR ACKNOWLEDGEMENTS}

No conflict of interest.

\section{PSQ-140 THE COMMUNICATION PROCESS BETWEEN PHARMACY AND OTHER DEPARTMENTS AND WARDS IN AN ACUTE HOSPITAL}

N Kilcullen*. Tallaght University Hospital, Pharmacy, Dublin, Ireland

10.1136/ejhpharm-2019-eahpconf.573

Background The pharmacy department communicates information by emailing memos to clinical staff. Paper copies are also distributed to clinical areas. Previous research highlighted poor awareness of information distributed via these channels.

Purpose The aim of the project was to examine current communication methods, explore alternative methods and to improve the effectiveness of communication between the pharmacy and other departments and wards.

Material and methods A pharmacist-led multi-disciplinary team, including pharmacists, pharmaceutical technicians, clinical nurse managers (CNMs), a non-consultant hospital doctor (NCHD) and a dietician, carried out a Quality Improvement project.

Voice of the Customer (VOC) - Critical to Quality (CTQ) and CTQ-Measure Quality Improvement tools were used to develop an 18-question survey. Question categories included:

- Current process questions e.g. 'Where do you currently look for pharmacy information?'.

- Knowledge of information disseminated in recent memos.

- Barriers to receiving pharmacy information.

- Preferred means of receiving pharmacy information.

Sixty staff (18 NCHDs, 20 nurses, 14 CNMs, eight dieticians) were asked to complete the survey: the response rate was $100 \%$. Cause and Effect analysis was carried out to identify factors leading to communication problems. Based on the findings, alternative communication techniques were proposed and piloted over a 2 week period.

Results Significant variation in how memos were displayed on wards was evident.

Seventy per cent of nurses surveyed checked their emails once-weekly or less frequently, indicating that this is not an effective method of communication.

Twenty-five per cent of nurses surveyed and $17 \%$ of NCHDs surveyed were aware of the contents of a recent pharmacy memo.

Respondents in all categories indicated a preference for verbal communication of pharmacy information.

Pilot results Memos were displayed on wards in a display folder known as the 'Pharmacy Communication Hub'. Awareness of recent memos increased from $25 \%$ to $69 \%$ of nurses surveyed.

Verbal communication of urgent memos by pharmacists to NCHDs was piloted. Memo awareness among NCHDs increased from $17 \%$ to $87.5 \%$ of NCHDs surveyed.

Conclusion This project found that existing pharmacy communication techniques were not effective. Alternative communication methods were piloted and demonstrated improved effectiveness. Implementation of these methods will ensure that up-to-date medication-related information is both highlighted to, and is easily accessible by, clinical staff.

\section{REFERENCES AND/OR ACKNOWLEDGEMENTS}

Project Team. Nurse Practice Development.

No conflict of interest.

\section{PSQ-141 POLYPHARMACY AND TRANSCATHETER AORTIC VALVE IMPLANTATION}

${ }^{1} \mathrm{G}$ Lo Surdo*, 'E Volpi, 'M Zizevskikh, 'S Tonazzini, 'S Alduini, ${ }^{2} \mathrm{~A}$ Mazzone, ${ }^{3} \mathrm{~S}$ Forni, ${ }^{3} \mathrm{~S}$ D'Arienzo, ${ }^{4} \mathrm{M}$ Baroni, ${ }^{1} \mathrm{~S}$ Biagini. ${ }^{1}$ Fondazione Toscana Gabriele Monasterio, Hospital Pharmacy, Massa, Italy; ${ }^{2}$ Fondazione Toscana Gabriele Monasterio, Division of Clinical and Surgical Heart Diseases, Massa, Italy; ${ }^{3}$ Regional Health Agency, Quality Unit, Firenze, Italy;

${ }^{4}$ Fondazione Toscana Gabriele Monasterio, Clinical Risk Manager, Massa, Italy

10.1136/ejhpharm-2019-eahpconf.574 